Nevşehir Bilim ve Teknoloji Dergisi Cilt 6(1) 1-9 2017

DOI: 10.17100/nevbiltek.313368

URL: http://dx.doi.org/10.17100/nevbiltek.313368

\title{
Gaussyen Potansiyel İle Sınırlandırılmış Tek Elektronlu Kuantum Noktanın İstatistiksel Mekaniksel Modellenmesi ve Termodinamik Özellikleri
}

\author{
Gorkem OYLUMLUOGLU ${ }^{1, *}$ \\ ${ }^{1}$ Mugla Sitki Kocman Üniversitesi, Fen Fakültesi, Fizik Bölümü, Mugla
}

Öz

$\mathrm{Bu}$ çalışmada, Gaussyen potansiyel ile sınırlandırılmış GaAs kuantum noktanın istatistiksel mekaniksel modellenmesi ve termodinamik özellikleri araştırılmıştır. Manyetik alan içerisindeki parçacığın kutupsal koordinatlardaki Hamiltoniyeni çözülmüş, enerji özdeğer ve özdurumları bulunmuştur. Fock Darwin durumları olan bu öz durumlar kullanılarak pertürbasyon teorisi yardımıyla sistemin toplam enerjisi elde edilmiştir. Böylelikle kanonik topluluk yaklaşımı ile elde edilen partisyon fonksiyonundan; serbest enerji, entropi, ortalama enerji ve 1sı kapasitesi gibi termodinamik niceliklerin değişimleri, sıcaklık, manyetik alan, Gaussyen potansiyelin erimi ve Gaussyen potansiyelin derinliği gibi parametrelere bağlı olarak incelenmiştir.

Anahtar Kelimeler: Düşük Boyutlu Sistemler, Kuantum Nokta, Gaussyen Potansiyel.

\section{Statistical Mechanical Modelling and Termodynamical Properties of Single Electron Quantum Dot with Gaussian Confinement}

\begin{abstract}
In this study, statistical mechanical modelling and termodynamical properties of a GaAs quantum dot with Gaussian confinement have been investigated. The Hamiltonian of a particle under magnetic field was solved in polar coordinates, energy eigenvalues and eigenfunctions have been obtained. The total energy of the system was found by using the Fock Darwin states and the first order Perturbation theory. Therefore, the behavior of the thermodynamical quantities of the system such as, free energy, entropy, average energy and heat capacity were investigated as a function of temperature, magnetic field, quantum dot size and Gaussian potential depth by using the partition function obtained by using canonical ensemble approach.
\end{abstract}

Keywords: Low Dimensional Systems, Quantum Dot, Gaussian Potential.

*e-mail: gorkem@mu.edu.tr 


\section{Introduction}

In recent years, quantum dots have become one of the important, prominent topics of condensed matter physics. This can be explained in two ways: the first of them is application area of quantum dots with promising application potential; for instance, solar cells, single electron transistors, quantum computers and microelectronic applications such as quantum lasers. The second and less important one is quantum dots are small laboratories where quantum mechanics can be tested.

For solving to any quantum problem, we must first understand the nature of the confinement potential. After the first experiments, it is realized that these potentials are harmonic. However, in recent experiments, it has been shown that, the confinement potential is non-harmonic and should be a finite depth. In this context, Gaussian potential is an important candidate for confinement potential and there have been many studies on this in recent years [1]. These studies are the researches for learning many properties of quantum dots, such as electronic, magnetic and thermodynamic properties, in single-particle and multi-particle situations.

In semiconductor physics, there are structures which limit the three dimensions of electrons and carry them to two dimensions, it is called 'Quantum Wells', and the other structures which electrons can move in one dimension are called 'Quantum Wires [2-5]. In recent years, Adamowski and his colleagues investigate the properties of excited electrons in quantum dots, depends on the attractive Gaussian potential which limits this quantum dot [1]. This potential is at a finite depth, exhibiting parabolic features at the center of the quantum dot and providing the generalized Kohn theorem [6]. Various calculation methods have been used while physical properties of quantum dot structures are investigated, these are Perturbation Methods and Variational Methods. At spherical quantum dot, when the impurity atom is outside the center of the sphere, the binding energy is calculated by the Perturbation method [6,7]. In the last few years, in the presence of the magnetic field, many discoveries have been made about the heat capacity and entropy of quantum dots and the other related low- dimensional systems [9-12]. In addition, most of the investigations were made for square potential wells or confined parabolic potential quantum wells. As a result of these calculations, peaks were observed in energy levels in heat capacity and sustainability. Oh. et al. (1994) has calculated the heat capacitance in quantum wires and quantum dots and have observed heat capacitance which exhibits oscillation behavior at low temperatures [6,10]. Also they have researched magnetization, magnetic sustainability and heat capacity in helium-like confined quantum dots and have introduced additional structures for heat capacitance [9].

Maksym and Chakraborty investigated electronic heat capacity by taking account electron interactions [6,9]. All calculations are made by using parabolic confinement potential. According to our knowledge ever, in the presence of spin Zeeman interactions, there is not enough scientific research done in temperature-dependent heat capacity and entropy in Gaussian potentially confined quantum dots.

\section{Material and Methodology}

Nowadays, making new technics by taking advantage of the physical properties of one and zero dimensional semiconductor systems are among the most popular topics. The shapes, dimensions, energy levels, and the number of electrons of quantum dot structures, where electron motion is limited to all dimensions, can be controlled. Hence, it is attracted a great deal of attention technologically. Hamiltonian 
of an electronic system, moving in two dimensions, limited to $V$ potential and in the presence of Hamiltonian of an electronic system, moving in two dimensions, limited to $V$ potential and in the presence of $\vec{B}$ external magnetic field;

$$
H=\frac{1}{2 m^{*}}\left(\vec{p}+\frac{e}{c} \vec{A}\right)^{2}+V(\rho)
$$

can be written as above. After some arrangements on Hamiltonian:

$$
H=-\frac{\hbar^{2}}{2 m^{*}} \nabla^{2}+\frac{1}{2} \omega_{c} L_{z}+\frac{1}{8} m^{*} \omega_{c}^{2} \rho^{2}+V(\rho)
$$

we can obtain relation above. Where $\rho$ is the position of electron moving in two dimensions, $\hat{p}$ is the momentum operator, $m^{*}$ is effective mass, and $\vec{A}$ is vector potential corresponding to the magnetic field $\mathrm{B}$ applied in the z-direction. And $\omega_{c}=\frac{e B}{m^{*}}$ where frequency of cyclotron, $B$ is magnetic field and $V(\rho)=$ $-V_{0} e^{-\frac{\rho^{2}}{2 R^{2}}}$ represents confinement potential. $V_{0}$ is the depth of potential, $R$ is width of potential, and so represents size of quantum dots and effective confinement length respectively.

Where; $\omega_{h}=\frac{V_{0}}{m^{*} R^{2}}$ ve $\widetilde{\omega}^{2}=\frac{1}{4} \omega_{c}^{2}+\omega_{h}^{2}$. Term of $\lambda$ to be a key term in a model; if $\lambda=0$ is taken Harmonic Potential, if $\lambda=1$ is taken Gaussian Potential is represented by $\lambda$. When Eq.2 is derived according to above relation;

$$
H=-\frac{\hbar^{2}}{2 m^{*}} \nabla^{2}+\frac{1}{2} \omega_{c} L_{z}+\frac{1}{2} m^{*} \rho^{2} \widetilde{\omega}^{2}-V_{0}-\lambda\left[\frac{1}{2} m^{*} \omega_{h}^{2} \rho^{2}+V_{0}\left(e^{-\frac{\rho^{2}}{2 R^{2}}}-1\right)\right]
$$

where $\mathrm{H}$ is the identify whole of the system Hamiltonian operator, and there is completely solvable $H_{0}$, and where $H_{1}$ makes the solution difficult but with a small contribution to the eigenvalues and eigenfunction of $H_{0}$, if it is written form of the sum of $H_{1}$ and is solved by Perturbation Theory;

$$
H=-\frac{\hbar^{2}}{2 m^{*}} \nabla^{2}+\frac{1}{2} \omega_{c} L_{z}-V_{0}+\frac{1}{2} m^{*} \rho^{2}\left[(1-\lambda) \omega_{h}^{2}+\frac{1}{4} \omega_{c}^{2}+\frac{4 \lambda V_{0} \omega_{h o}}{\left(\hbar+4 R^{2} m^{*} \omega_{h o}\right)}\right]
$$

we can obtain solution above. Where $\omega^{2}=m^{*} \rho^{2}\left[(1-\lambda) \omega_{h}^{2}+\frac{1}{4} \omega_{c}^{2}+\frac{4 \lambda V_{0} \omega_{h o}}{\left(\hbar+4 R^{2} m^{*} \omega_{h o}\right)}\right]$;

$$
H=-\frac{\hbar^{2}}{2 m^{*}} \nabla^{2}+\frac{1}{2} \omega_{c} L_{z}-V_{0}+\frac{1}{2} \omega^{2}
$$

we obtain above relations.

Following Hamiltonian solution is the form of Fock Darwin

$$
H \Phi_{n l}(\rho)=E_{n l} \Phi_{n l}(\rho)
$$


Where $\Phi_{n l}(\rho)$ Fock Darwin wavefunction;

$$
\Phi_{n l}(\rho)=\frac{\alpha}{\sqrt{\pi}}\left(\frac{n !}{(n+|l|) !}\right)^{2}(\alpha \rho)^{|l|} L_{n}^{|l|}\left(\alpha^{2} \rho^{2}\right) e^{-\frac{1}{2} \alpha^{2} \rho^{2}+i l \theta}
$$

we have $\alpha=\sqrt{\frac{m^{*} \omega}{\hbar}}$.

The energy eigenvalues of this wave function;

$$
E_{n l}=(2 n+|l|+1) \hbar \omega+\frac{1}{2}|l| \hbar \omega_{c}-V_{0}
$$

as expected. Where $n$ is Radial quantum number and $l$; Azimuth angular momentum quantum number.

Modelling by using physical properties of Gaussian confined potential GaAs quantum dots and canonical distribution when examining physical properties by statistical mechanical methods;

$$
Z=\sum_{n, l} e^{-\frac{E_{n l}}{k_{B} T}}
$$

defined as above. When the ground state energy is substituted in Equation 8 and Equation 9;

$$
Z\left(T, B, V_{0}, R\right)=\sum_{n=0}^{\infty} \sum_{l=0}^{ \pm \infty} e^{-\frac{\left[(2 n+|l|+1) \hbar \omega+\frac{1}{2}|l| \hbar \omega_{c}-V_{0}\right]}{k_{B} T}}
$$

Partition function of system;

$$
Z\left(T, B, V_{0}, R\right)=\frac{\operatorname{cosch}\left(\frac{g \hbar \omega_{c}}{4 k_{B} T}\right)}{\cosh \left(\frac{\hbar \omega}{k_{B} T}\right)-\operatorname{cosch}\left(\frac{\hbar \omega_{c}}{2 k_{B} T}\right)}
$$

obtained as above.

\section{Results}

In this study, the physical properties of Gaussian confined potential GaAs quantum dots are modelled and ground state energy is obtained using Perturbation theory. Also partition function and phenomenon was obtained using Boltzmann Gibbs Statistics was understood in microscopic level, and was calculated all other thermodynamics sizes. In this model, in the presence of the Zeeman term, the thermodynamic properties of confined Gaussian potential GaAs quantum dots were investigated with respect to heat capacity and entropy changes depending on different parameters. These parameters are: 
temperature, magnetic field, range of Gaussian potential, effective potential and depth of Gaussian potential.

Free Energy;

$$
F\left(T, B, V_{0}, R\right)=-k_{B} T \ln Z\left(T, B, V_{0}, R\right)=-k_{B} T \ln \frac{\operatorname{cosch}\left(\frac{g \hbar \omega_{c}}{4 k_{B} T}\right)}{\cosh \left(\frac{\hbar \omega}{k_{B} T}\right)-\operatorname{cosch}\left(\frac{\hbar \omega_{c}}{2 k_{B} T}\right)}
$$

is derived as above.

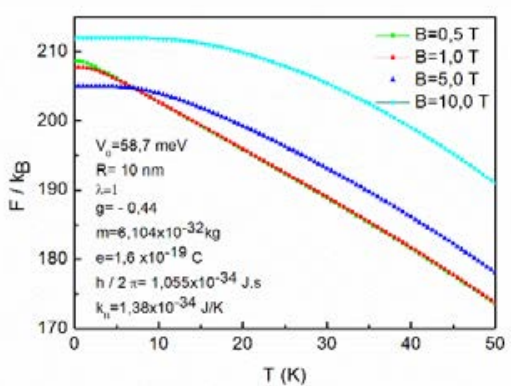

(a)

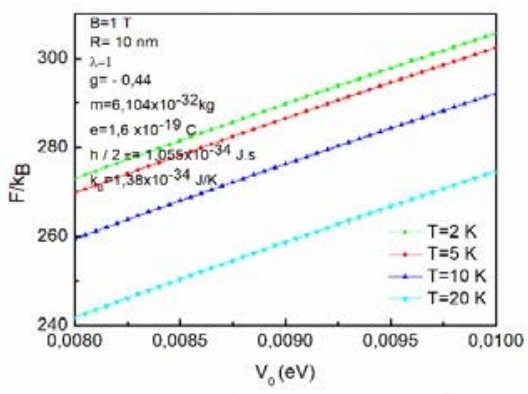

(c)

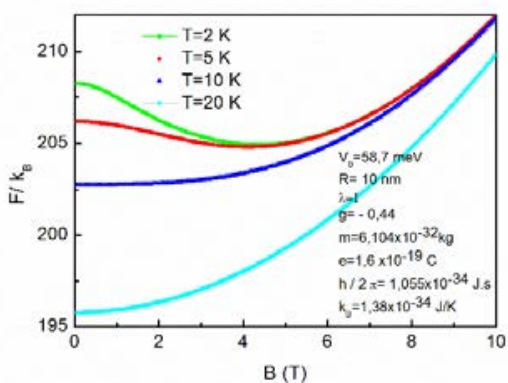

(b)

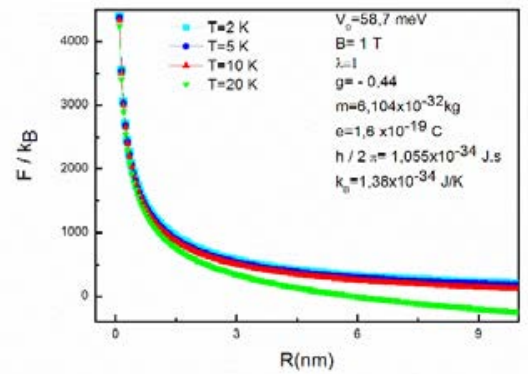

(d)

Figure 1. a) Temperature dependent free energy. b) Magnetic Field dependent free energy. c) Potential Depth dependent free energy. d) Width of quantum dot dependent free energy.

In Figure 1a, Temperature dependent free energy is given for different magnetic fields. Free energy decreases depend on the temperature and increase with magnetic field increasing proportionally. Free energy, decreases by depending on the temperature and increases with magnetic field increasing. In Figure $1 \mathrm{~b}$, the variation of the free energy against the magnetic field is given for different temperatures. Free energy or free enthalpy increases due to magnetic field and inversely proportionally decreases with increasing in temperature. At low values of magnetic fields, due to increasing in kinetic energy efficiency which causes of electron trapping, free energy increases due to temperature at low magnetic field values. The graph of free energy versus potential depth is given in Figure 1c. As the potential depth increases, the free energy increases monotonically. It is observed that, free energy decreases somewhat due to changing in temperature. In Figure 1d, the free energy is plotted for different temperatures depending on the width 
of the quantum dot. It has been found that, depends on size of quantum dots, free energy exhibits exponential behaviors at high temperatures.

Average energy;

$$
\begin{aligned}
E\left(T, B, V_{0}, R\right)= & -\frac{\partial}{\partial T} \ln Z\left(T, B, V_{0}, R\right) \\
= & \frac{\hbar}{4}\left[\frac{4 \omega \sinh \left(\frac{\hbar \omega}{k_{B} T}\right)-2 \omega_{c} \sinh \left(\frac{\hbar \omega_{c}}{2 k_{B} T}\right)}{\cosh \left(\frac{\hbar \omega}{k_{B} T}\right)-\cosh \left(\frac{\hbar \omega_{c}}{2 k_{B} T}\right)}-g \omega_{c} \tanh \left(\frac{g \hbar \omega_{c}}{4 k_{B} T}\right)\right]
\end{aligned}
$$

is obtained as above derivation.

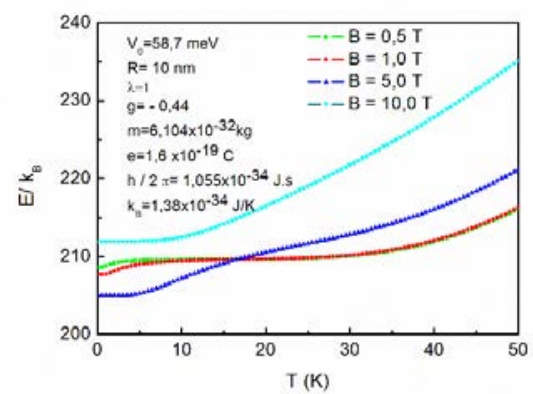

(a)

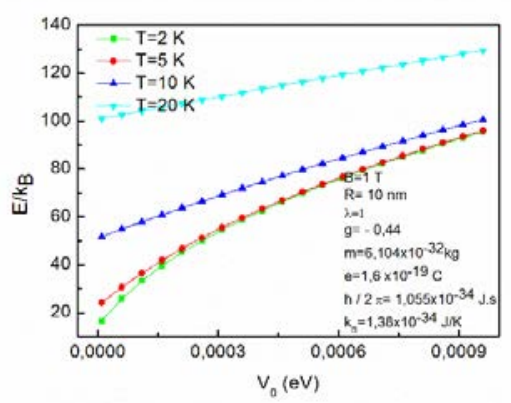

(c)

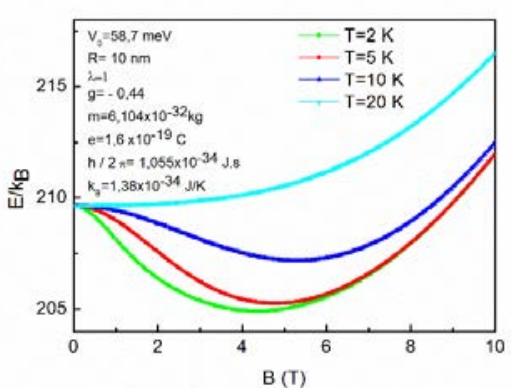

(b)

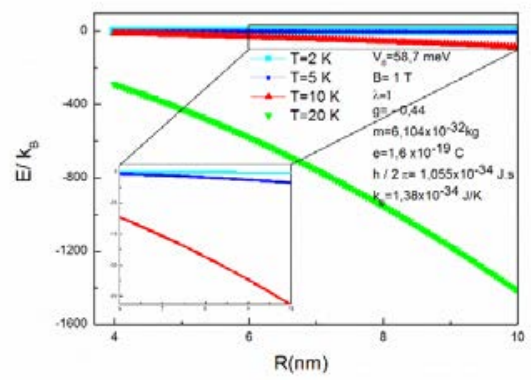

(d)

Figure 2. a) Temperature dependent average energy b) Magnetic Field dependent average energy. c) Potential Depth dependent average energy. d) Width of quantum dot dependent average energy.

As seen in Figure 2a, average energy increases exponentially depending on the temperature for all magnetic field values. In figure $2 \mathrm{~b}$, magnetic field versus average energy graph is plotted. At low temperatures, spin effects cause decreasing by the increasing in magnetic field initially, and then monotone increasing is observed. But, monotone increasing only is observed at high temperatures. In figure 2c, as the depth of the well increases, the average energy increases as expected. Similarly, if temperature will increase, also average energy will increase proportionally. So the calculations are made in line with the expected results. In figure $2 \mathrm{~d}$, the graph of average energy versus change due to the width of quantum dot is plotted for different temperatures. At low temperatures, the average energy has not changed, but at high temperatures (over 100K) a sharp decline is observed. 
Heat capacitance;

$$
\begin{aligned}
& C_{v}\left(T, B, V_{0}, R\right)=-T \frac{\partial^{2} F\left(T, B, V_{0}, R\right)}{\partial T^{2}} \\
& =\frac{\hbar^{2}\left\{\begin{array}{c}
\left.-2\left(4 \omega^{2}+\omega_{c}^{2}\right)+\left(2 \omega+\omega_{c}\right)^{2} \cosh \left[\frac{\hbar\left(2 \omega+\omega_{c}\right)}{2 k_{B} T}\right]+\left(-2 \omega+\omega_{c}\right)^{2} \cosh \left[\frac{\hbar\left(2 \omega+\omega_{c}\right)}{2 k_{B} T}\right]+\right\} \\
\frac{g^{2}}{2} \omega_{c}^{2}\left[\cosh \left(\frac{\hbar \omega}{k_{B} T}\right)-\cosh \left(\frac{\hbar \omega_{c}}{2 k_{B} T}\right)\right]^{2} \operatorname{sech}^{2}\left(\frac{g \hbar \omega_{c}}{4 k_{B} T}\right)
\end{array}\right\}}{\left\{8 k_{B} T^{2}\left[\cosh \left(\frac{\hbar \omega}{k_{B} T}\right)-\cosh \left(\frac{\hbar \omega_{c}}{2 k_{B} T}\right)\right]^{2}\right\}}
\end{aligned}
$$

is obtained as Eq.14.

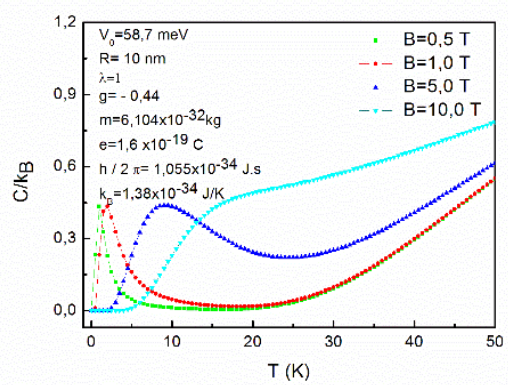

(a)

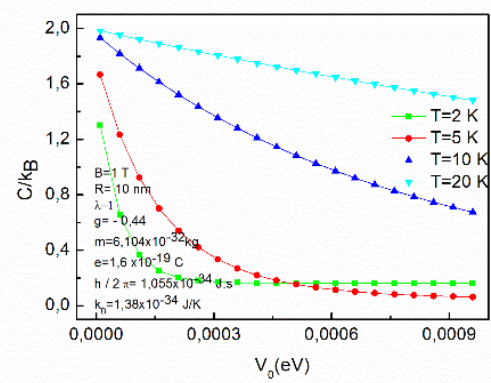

(c)

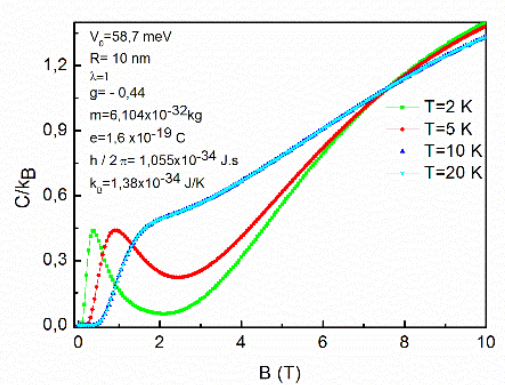

(b)

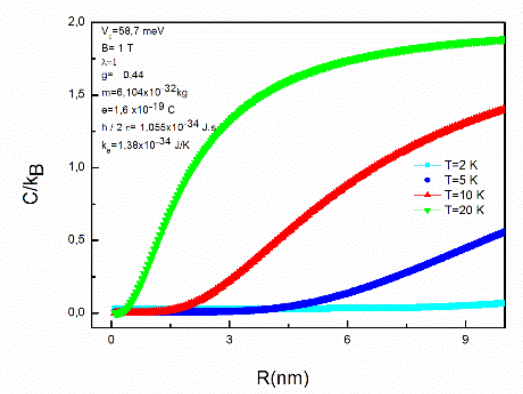

(d)

Figure 3. a) Temperature dependent heat capacity. b) Magnetic field dependent heat capacity. c) Potential depth dependent heat capacity. d) Width of quantum dot dependent heat capacity.

The temperature dependence of the heat capacity per electron is given in Figure 3a, taking into account the effect of electron spin. Peaks for different magnetic fields were observed at low temperatures. Unlike other magnetic fields, the $\mathrm{B}=10$ Tesla is shaped like a shoulder. When the magnetic field increases, the peaks disappear and the heat capacity approaches each other for all magnetic fields. These peaks are caused by the fluctuation in the spin states. If electrons are active at high temperatures, the effect of spin states is not observed. The dependence of the heat capacity for per electron on the magnetic field is given in Figure 3b, taking into account the effect of electron spin. At low magnetic fields, peaks were observed for different temperatures ( $2 \mathrm{~K}$ and $5 \mathrm{~K}$ ). Above $\mathrm{T}=10 \mathrm{~K}$, it takes the shape of a shoulder at a certain magnetic field value and the curves approach each other at high magnetic field values. Depending on the depth of the potential to trap the behavior of the heat capacity, the graph is given in Figure 3c. In the low values of $\mathrm{V}_{0}$, the curves intercept each other. This sharp decline is due to the large energy splitting between the lowest excited state and the ground state as $\mathrm{V}_{0}$ increases. Depending on the 
width of the quantum dot, the heat capacity is given in Figure 3d at different temperatures for 2 Tesla. The higher temperatures, the sharper change due to the width of the quantum dot. At $25 \mathrm{~K}$ and $50 \mathrm{~K}$, this behavior disappears as the temperature increases constantly over the range of the quantum dot.

\section{Discussion and Conclusion}

Quantum dots are quantum mechanical structures, also referred to as impurity atoms with atomic structure. These structures can be chemically synthesized, as well as can be designed of semi-conductor junction structures. In fact, these structures are restricted in three- dimensional quantum mechanically. This consideration allows to look at the problem as 'fermions in quantum well'. The adjustability of the size of the quantum well, enriches the using of these devices in technology which have atomic structures. Nowadays, there are technological application areas such as laser, single electron transistors, quantum computers with quantum byte in. There are many experimental studies on this subject that theoretical modeling is made both numerically and analytically. It is important to determine the thermodynamic and magnetic properties of the quantum dots with respect of their application areas.

Quantum dots are modeled using Gaussian potential. In general, parabolic potential well model is used in quantum dots. However, the knowledge that the potential exists at infinite depths of the parabolic potential is not enough to understand some of the features of quantum dots. Hence, the Gaussian potential well seems more realistic model, since it allows particles to break from these impurity atoms. Studies have shown that, the properties of the confined Gaussian potential quantum well and confined parabolic potential quantum well have shown similarity.

The determination of the thermodynamic properties of quantum dots is a significant way of understanding the device properties of this kind of systems. While, fluctuations and shoulder-like structures in the thermodynamic quantities of the quantum dot are observed at low temperature and magnetic fields, monotone changes are observed at high temperature and high magnetic fields. It is thought that these observations are caused by the spin of the particle. It is observed that the quantities of the changes which depends on potential depth are increased when the temperature increases. Furthermore, in the changes which depends on width of the quantum dots abnormal changes are observed above the certain temperature values.

\section{Acknowledgment}

The author is very grateful to Prof. Dr. Bahadir Boyacioglu (Vocational School of Health, Ankara University, 06290 Kecioren, Ankara, Turkey) for his useful comments and suggestions.

\section{References}

[1] Adamowski, J., Sobkowicz, M., Szafran, B. ve Bednarek, S., "Electron pair in a Gaussian confining potential”, Phys. Rev. B, 62, 4234-4240, 2000.

[2] Johnson, N.F., "Quantum dots: few-body, low-dimensional systems”, J. Phys. Condens. Matter, 7, 965-971, 1995.

[3] Woggon, U., “Optical Properties of Semiconductor Quantum Dots”, Springer, 230s, 1997.

[4] Jacak, L., Hawrylak, P. ve Wojs, A., “Quantum Dots”, Springer, 176 s, Germany, 1998. 
[5] Bimberg, D., Grundmann, M. ve Ledentsov, N.N., "Quantum Dot Hetero-structures", Wiley, 338s, Germany, 1998.

[6] Chatterjee, A., “1/N expansion for Gaussian potential”, J. Phys. A., 18, 2403-2422, 1985.

[7] Maksym, P.A. ve Chakraborty, T., "Quantum dots in a magnetic field: Role of electron-electron interactions”, Phys. Rev. Lett., 65, 108-122, 1990.

[8] Oh, J.H., Chang, K.J., Ihm, G. ve Lee, S., "Electronic structure of three-dimensional quantum dots in tilted magnetic fields”, J. Phys. Rev. B, 50, 15397-15410, 1994.

[9] Lee, S.J., Chang, J.H., Won. K.T., ve Souma, S., "Transitions of density of states and heat capacity in spherical quantum structure formed by a thin barrier”, Physica E, 40, 2198-2215, 2008.

[10] Gornik, E., Lassnig, R., Strasser, G., Steormer, H.L., Gossard, A. C. ve Wiegmann, W., "Specific Heat of Two-Dimensional Electrons in GaAs-GaAlAs Multilayers", Phys. Rev. Lett., 54, 1820-1832, 1985., 